\title{
A DUAL CCD MOSAIC CAMERA SYSTEM SEARCHING FOR MASSIVE COMPACT HALO OBJECTS (MACHOs)
}

\author{
Kem H. Cook \\ Lawrence Livermore National Lab
}

\begin{abstract}
The Macho Collaboration uses a dedicated 1.27-m telescope (The Great Melbourne Telescope) at Mount Stromlo to make photometric measurements of tens of millions of stars per night searching for the gravitational microlensing signature of MACHOs in the halo and disk of the Milky Way. A prime focus corrector and dichroic beamsplitter provide red $(6300-7800 \AA)$ and blue $(4500-6300 \AA)$ foci with one degree fields. A two by two mosaic of $2048 \times 2048$ pixel CCDs in each focal plane provides simultaneous images of 0.5 square degrees. By August of 1994, more than 20,000, 32 Megapixel images will have been collected of fields in the Large Magellanic Cloud (LMC), Small Magellanic Cloud and the bulge of the Milky Way. We have implemented an online analysis system which produces photometric reductions of a night's data (five Gbyte of images) within 24 hours. This system allows us to identify and follow interesting events in real-time. In this search, we have identified more than 60,000 variable stars, and a preliminary analysis of their types and distribution will be presented. Microlensing events toward the LMC and the bulge have been discovered and detection efficiencies are being calculated to constrain the MACHO content of the Milky Way's halo.
\end{abstract}

